\title{
Supporting Information: In Situ Nanoadjuvant-Assembled Tumor Vaccine for Preventing Long-Term Recurrence
}

\author{
Quoc-Viet Le', Juhan Suh ${ }^{1}$, Jinjoo Choi ${ }^{1}$, Gyu Thae Park ${ }^{1}$, Jung Weon Lee ${ }^{1,2,3}$, Gayong \\ Shim ${ }^{1, *}$ and $\mathrm{Yu}$-Kyoung $\mathrm{Oh}^{1, *}$ \\ ${ }^{1}$ College of Pharmacy and Research Institute of Pharmaceutical Sciences, ${ }^{2}$ Interdisciplinary \\ Program in Genetic Engineering, and ${ }^{3}$ Medicinal Bioconvergence Research Center, Seoul \\ National University, Seoul 08826, Republic of Korea
}

* Corresponding author (Tel: 82-2-880-2493; Fax: 82-2-882-2493)

E-mail addresses: shimg@snu.ac.kr (G. Shim), or ohyk@snu.ac.kr (Y.-K. Oh) 
Figures S1-S13

a

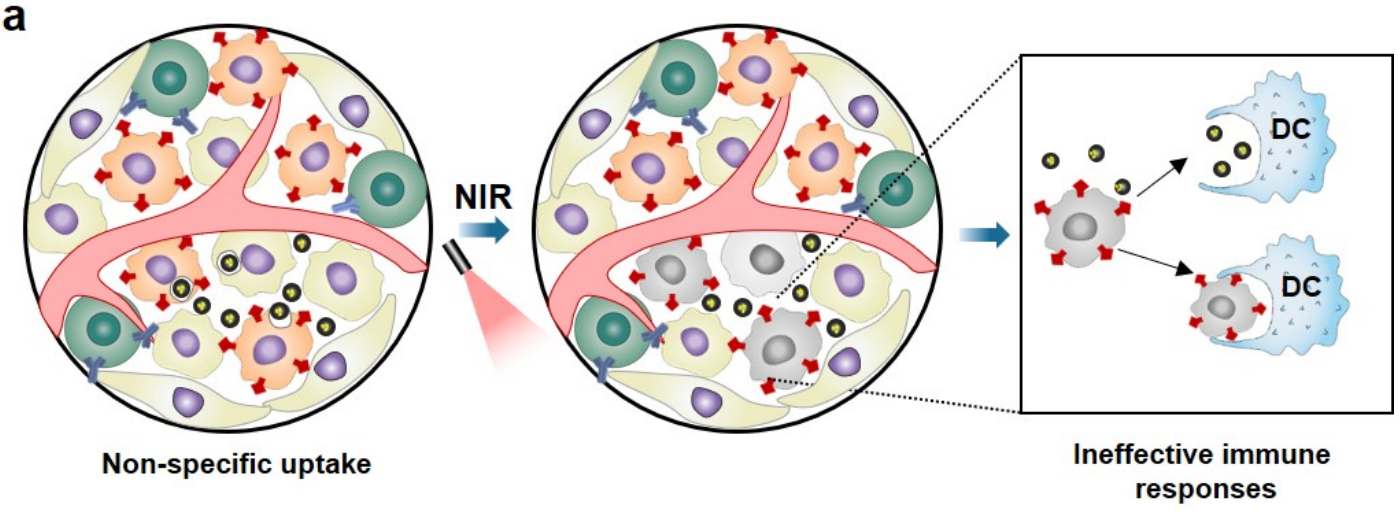

b

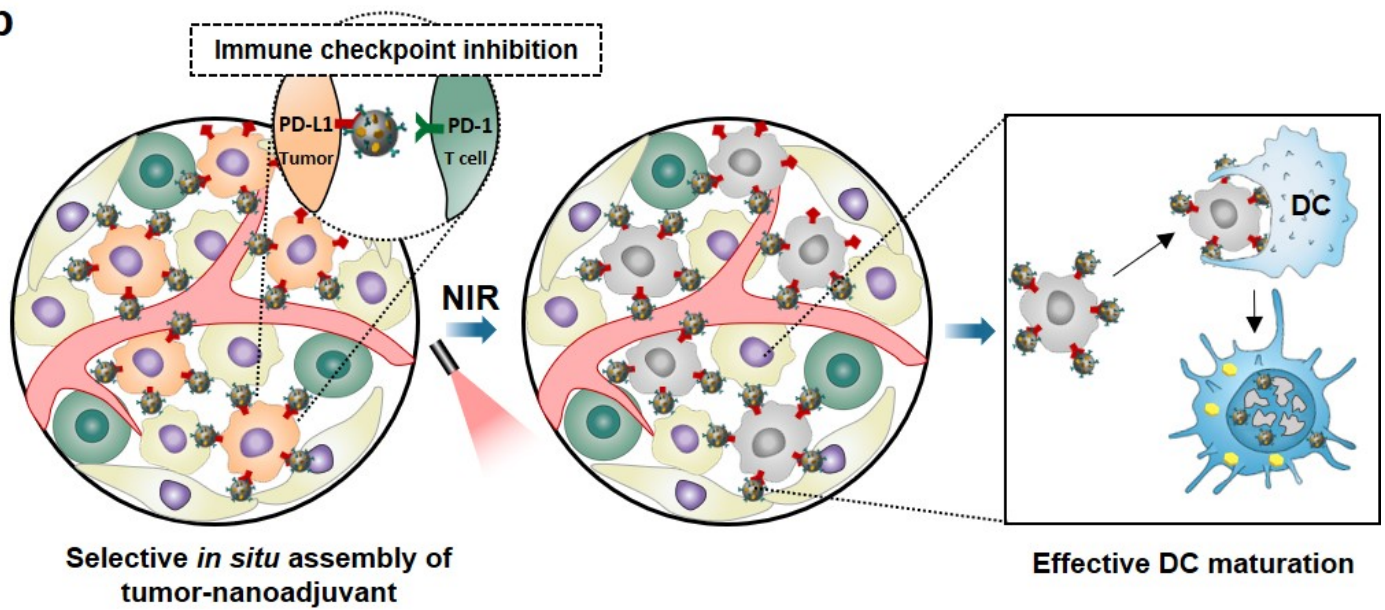

Figure S1. Advantages of in situ assembly of tumor and nanoadjuvants.

(a) Illustration of conventional combination of photothermal and immunotherapy. (b) Illustration of selective in situ assembly of tumor-nanoadjuvants and effective DC maturation upon NIR irradiation. 


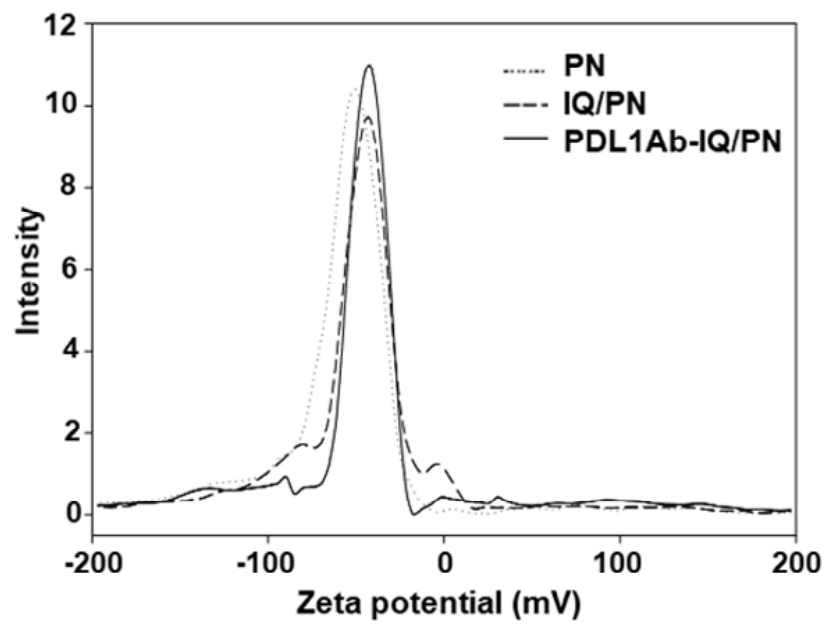

Figure S2. Zeta potential values of various nanoparticles.
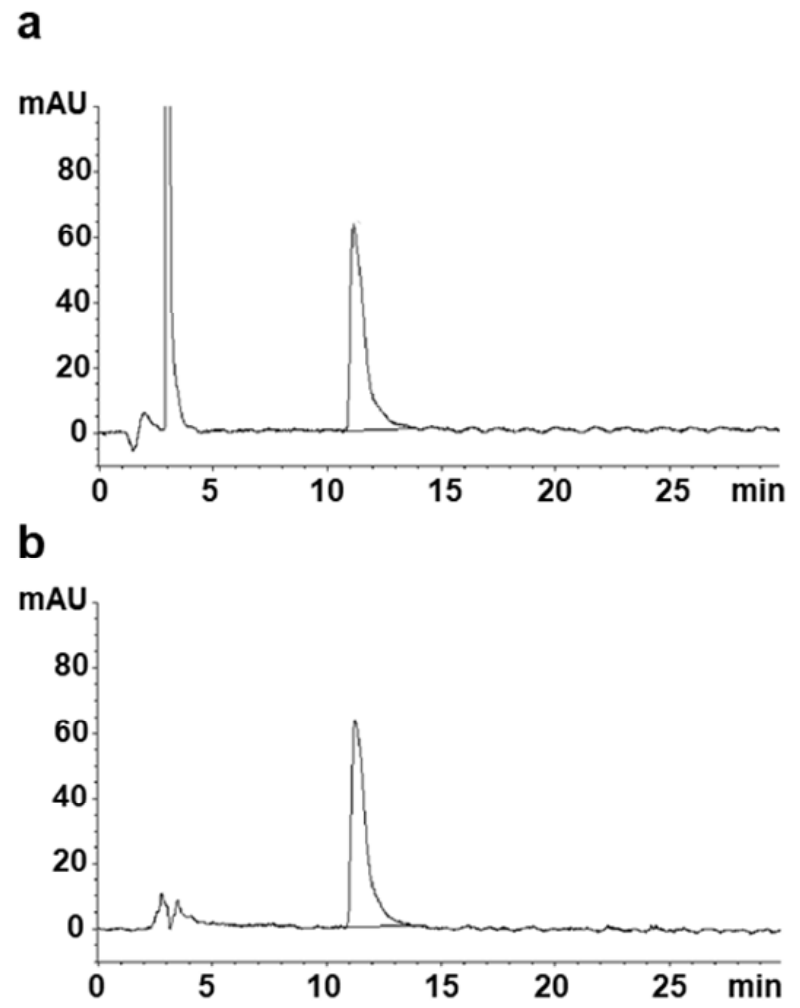

Figure S3. HPLC profile of IQ entrapped in PN.

IQ loading in PN was determined by measuring the amount of IQ by HPLC. HPLC profiles of IQ $(25 \mu \mathrm{g} / \mathrm{ml})$ in free form (A) and entrapped in PDL1Ab-IQ/PN (B). 

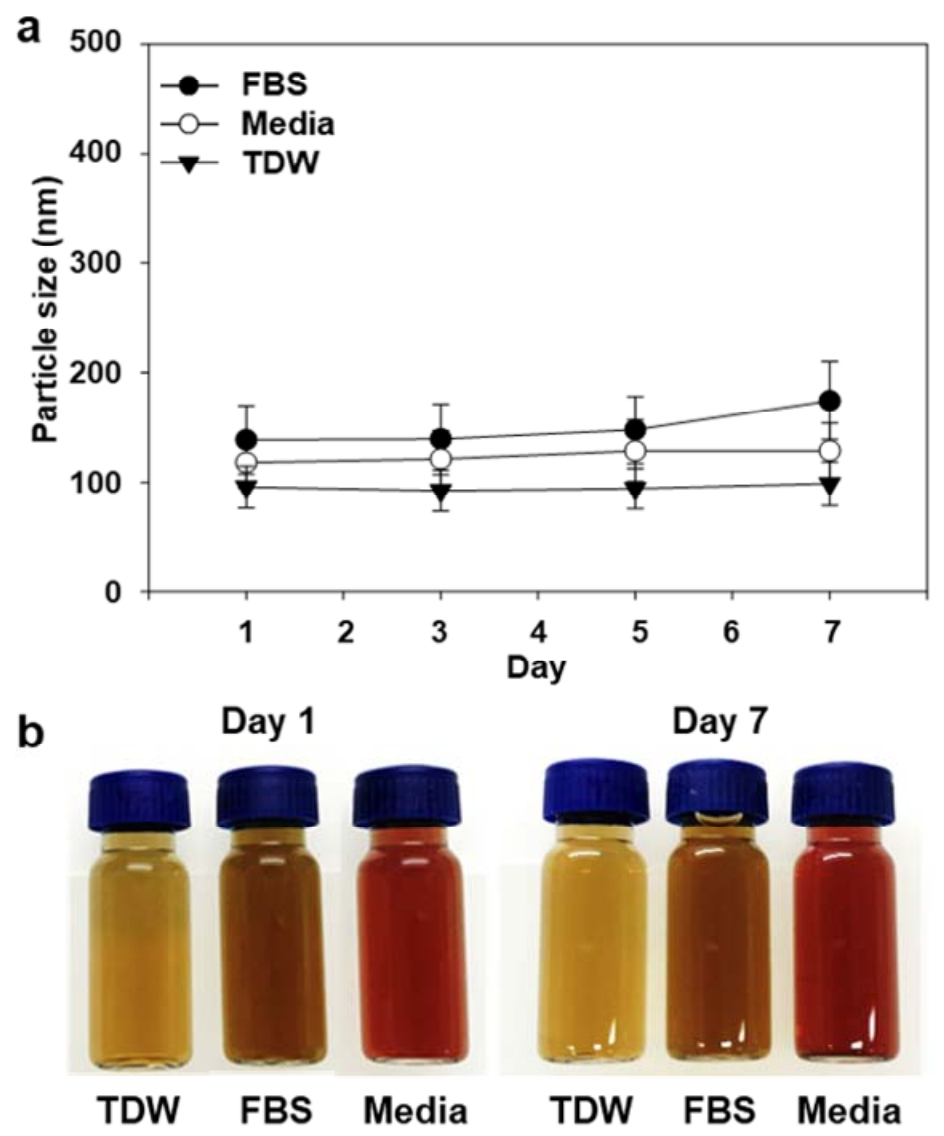

\section{Figure S4. Physical stability of PDL1Ab-IQ/PN.}

The dispersion stability of nanoparticles was evaluated over the course of 7 days in tripledistilled water (TDW), serum, and culture media. Particle size was measured by dynamic light scattering (a) on days 1, 3, 5 and 7, and the appearance (b) of PDL1Ab-IQ/PN samples was monitored visually on days 1 and 7 . 


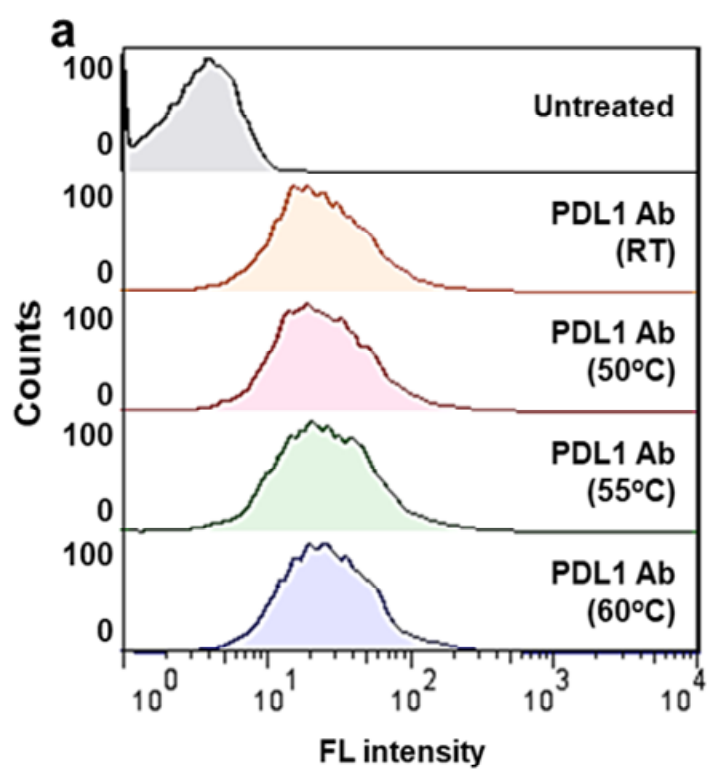

b
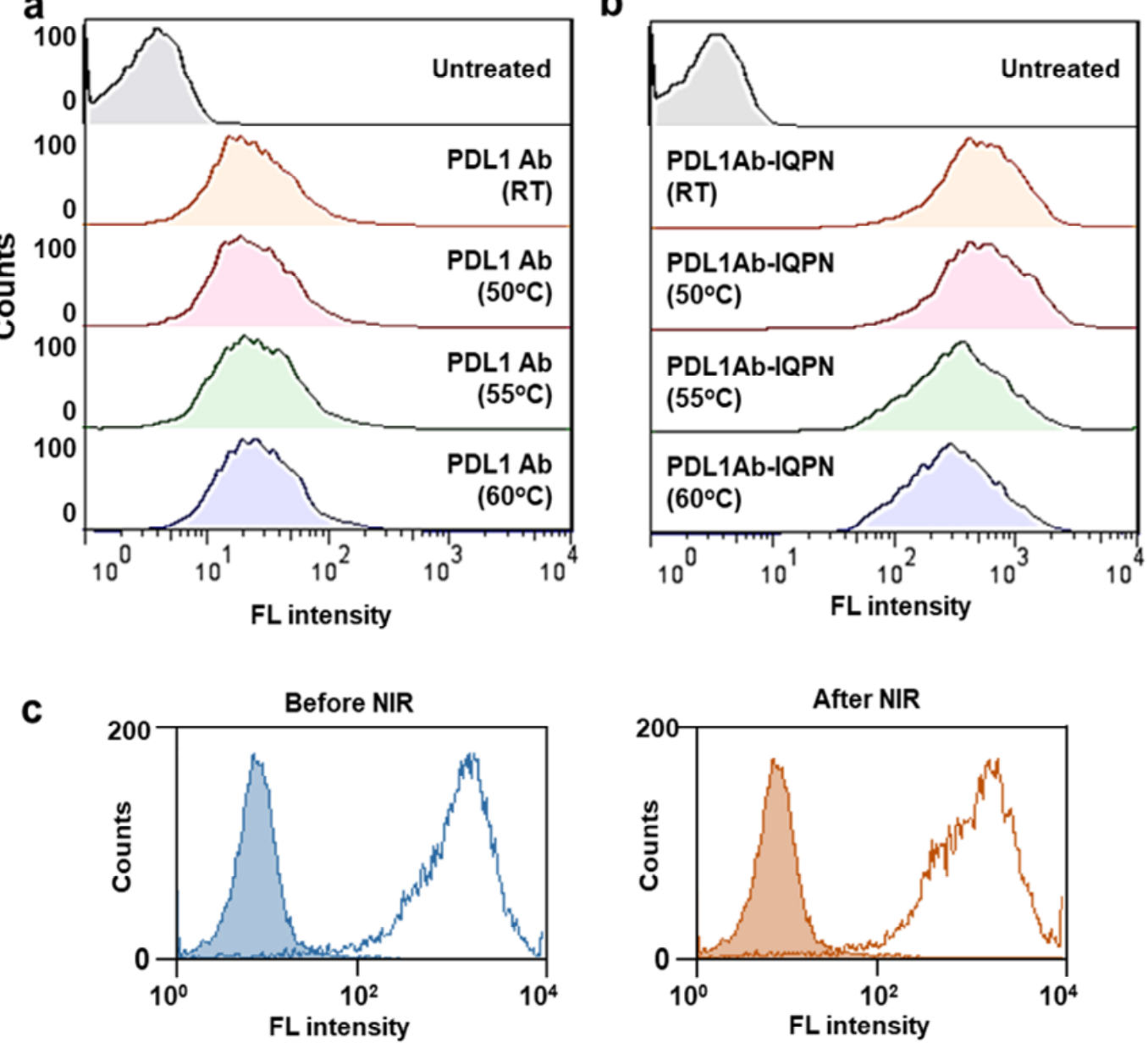

Figure S5. Thermal stability of PDL1Ab-IQ/PN.

PDL1Ab (a) or PDL1Ab-IQ/PNs (b) were heated for 5 min using a temperature-controlled heat block and then incubated with CT26 cells for $2 \mathrm{~h}$. The binding of PDL1Ab or PDL1Ab-IQ/PN to cells was measured by flow cytometry. (c) PDL1Ab-IQ/PN were NIR-irradiated with an $808 \mathrm{~nm}$ laser for $5 \mathrm{~min}$ to reach $60^{\circ} \mathrm{C}$, after which unirradiated or NIR-irradiated PDL1Ab-IQ/PN were incubated with CT26 cells for $2 \mathrm{~h}$. The binding of PDL1Ab-IQ/PN to cells was measured by flow cytometry. 
a

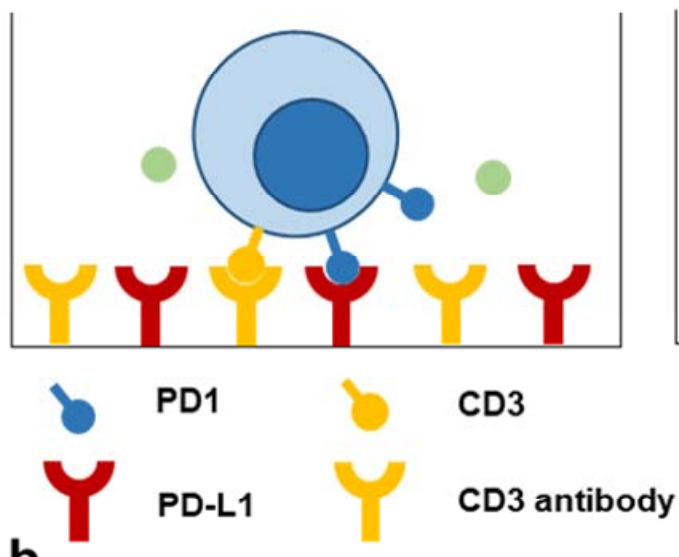

b

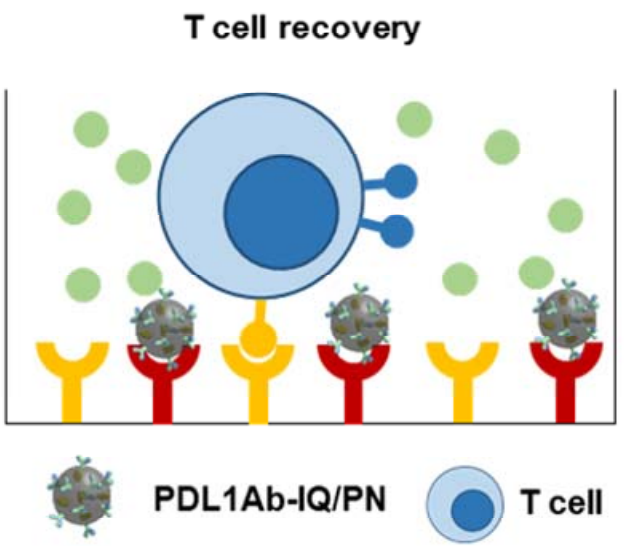

IL-2

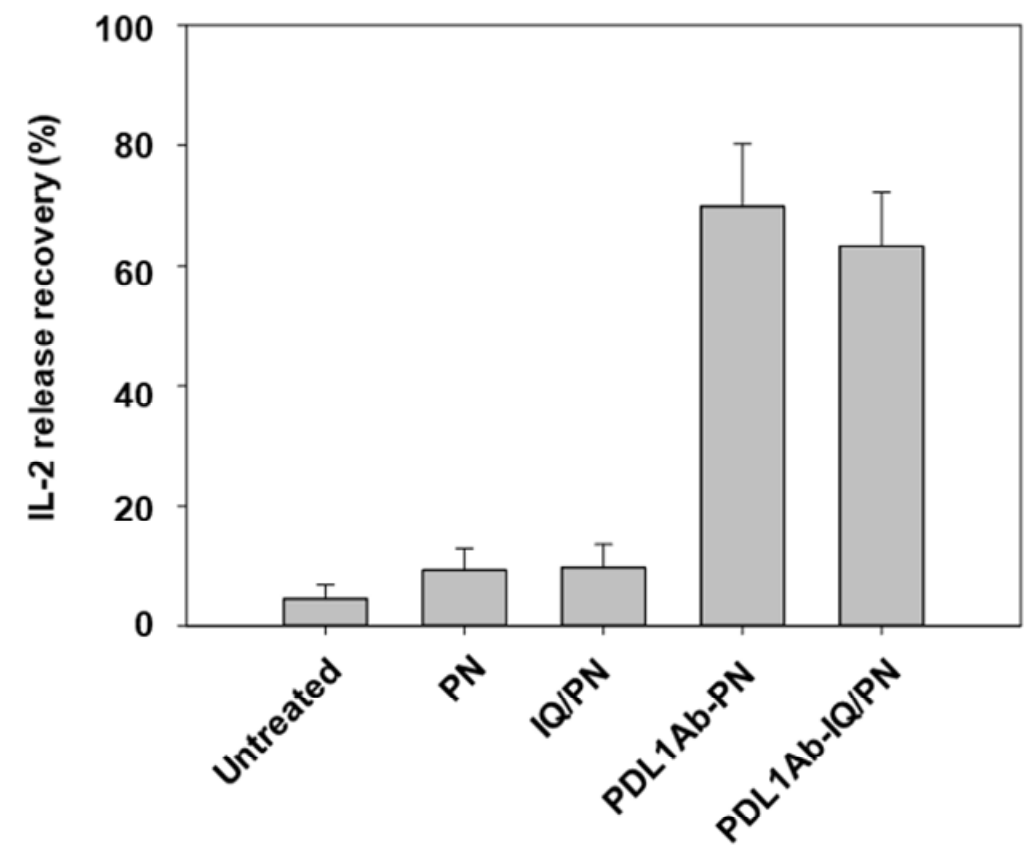

Figure S6. Immune checkpoint-blocking activity of PDL1Ab-IQ/PN.

(a) Illustration of the experimental design. The interaction of PDL1 with PD1 induces $\mathrm{T}$ cell suppression. In the presence of PDL1Ab-IQ/PN, inhibition of the immune checkpoint by $\mathrm{PDL} 1 \mathrm{Ab}-\mathrm{IQ} / \mathrm{PN}$ rescues the IL-2-release function of T cells. (b) PDL1/CD3 antibody-coated plates were incubated with IQ/PN or PDL1Ab-IQ/PN for $2 \mathrm{~h}$, after which $\mathrm{T}$ cells were added to the plate. After $48 \mathrm{~h}$, the levels of secreted IL-2 were measured by ELISA. 


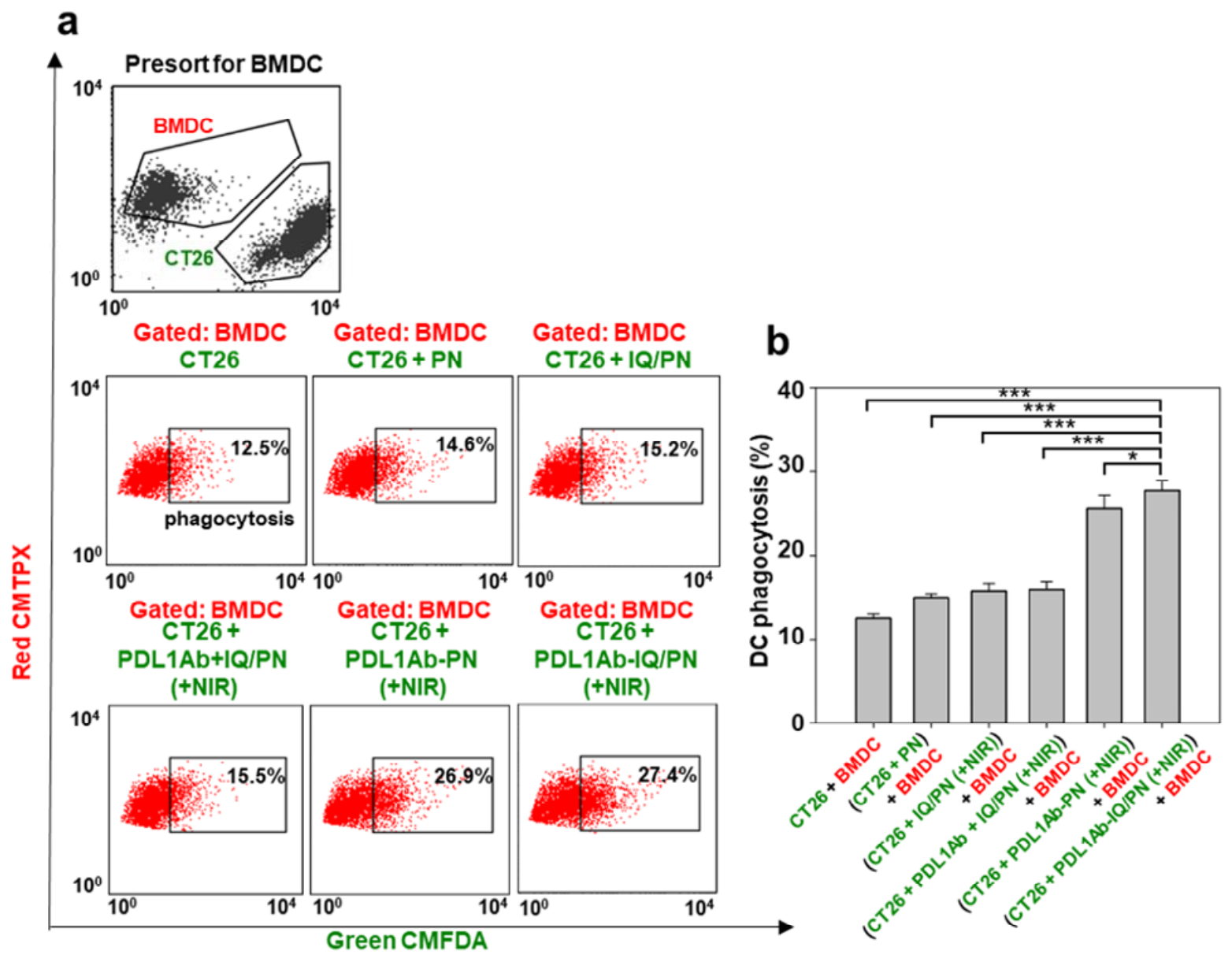

Figure S7. Phagocytosis of nanoformulation-treated tumor cells by DCs.

CT26 cells were treated with various nanoformulations, NIR-irradiated, and co-cultured with BMDCs. (a) Changes in fluorescence signals of BMDCs (red) were analyzed using flow cytometry. DC-phagocytosed CT26 cells (green) at $6 \mathrm{~h}$ of co-culture are indicated by black-lined boxes (a) and were quantified with BD CellQuest Pro software (b). ${ }^{*} P<0.05 ; * * * P<0.005$. 


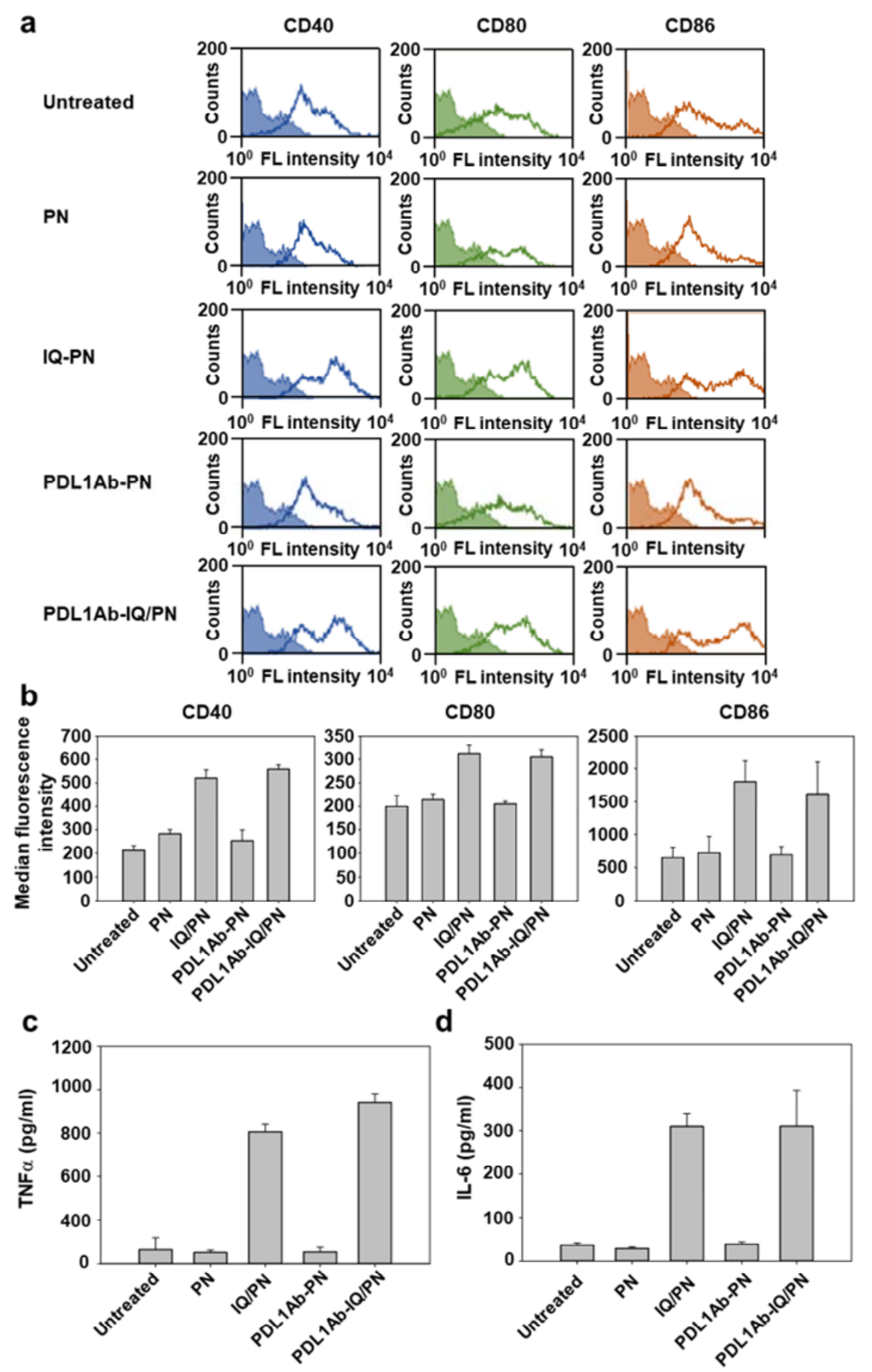

Figure S8. Induction of DC maturation with PDL1Ab-IQ/PN.

BMDCs were treated with various PNs. After 2 days, surface expression of CD40, CD80, and/or CD86 was confirmed by flow cytometry (a), and median fluorescence intensity was measured for quantification of antibody-positive cell populations (b). Secreted TNF- $\alpha$ (c) and IL-6 (d) were measured by ELISA. 
a
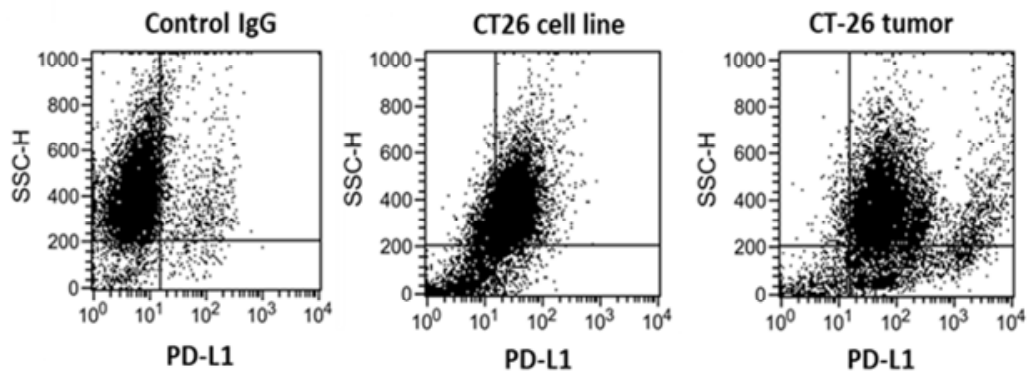

b

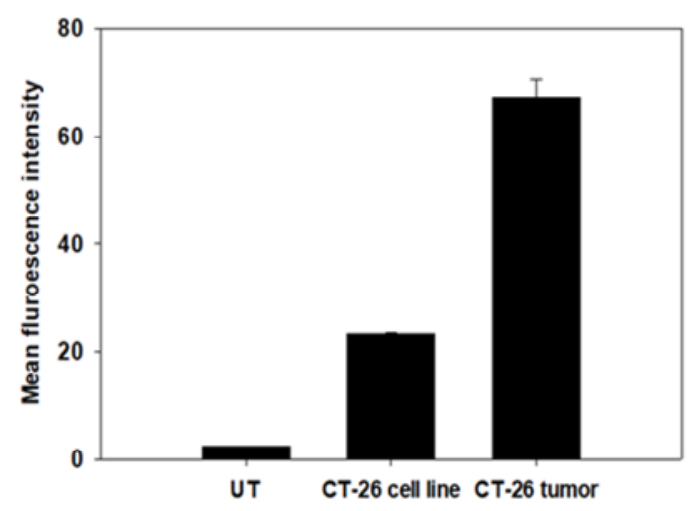

Figure S9. PDL1 expression on CT26 tumors.

Surface expression of PDL1 on CT26 cells was measured in cells cultured in vitro and cells isolated from in vivo tumor tissues. Expression levels were compared by flow cytometry (a), and mean intensity values of cells were quantified (b). 


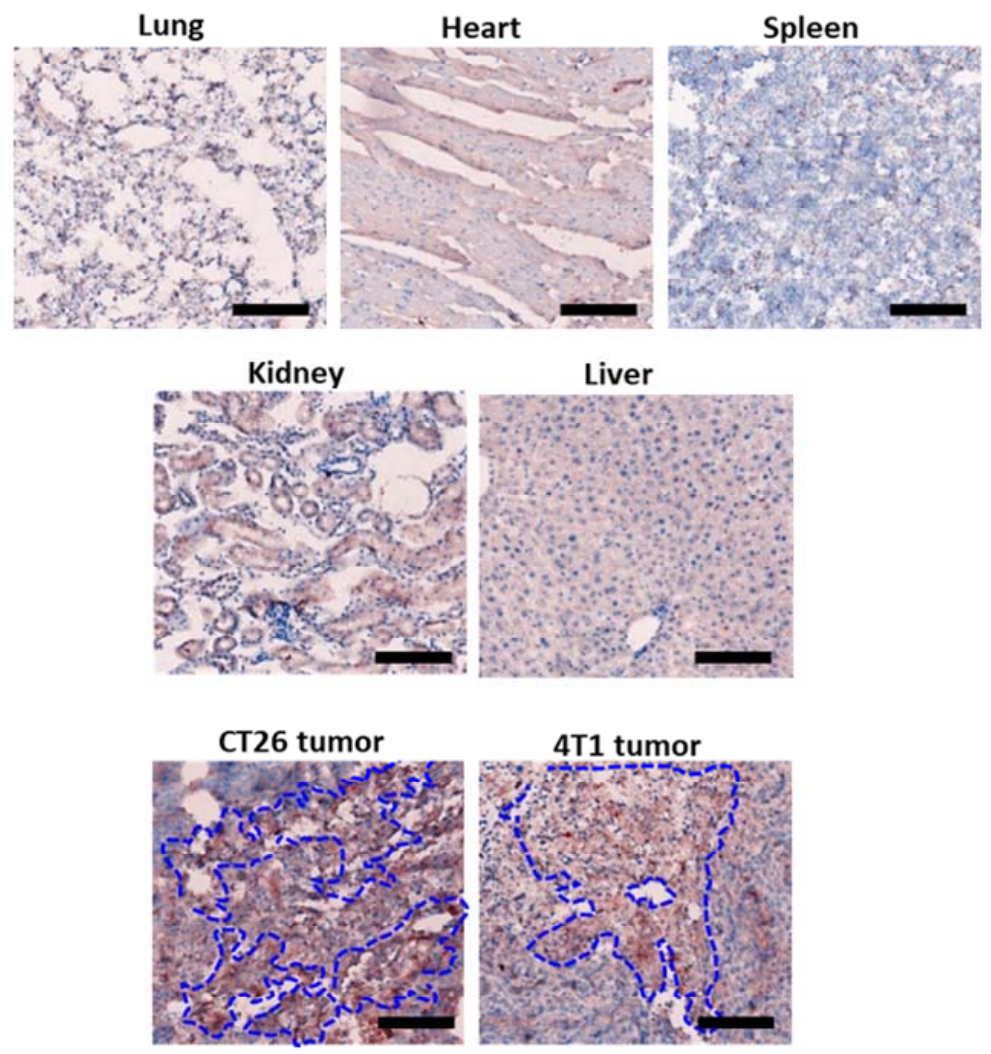

Figure S10. PDL1 expression in normal and tumor tissues.

Seven days after tumor inoculation, tumor tissue and main organs were immunohistochemically stained for PDL1. Scale bar: $100 \mu \mathrm{m}$. Blue dotted lines indicate PDL1-expressing areas. 


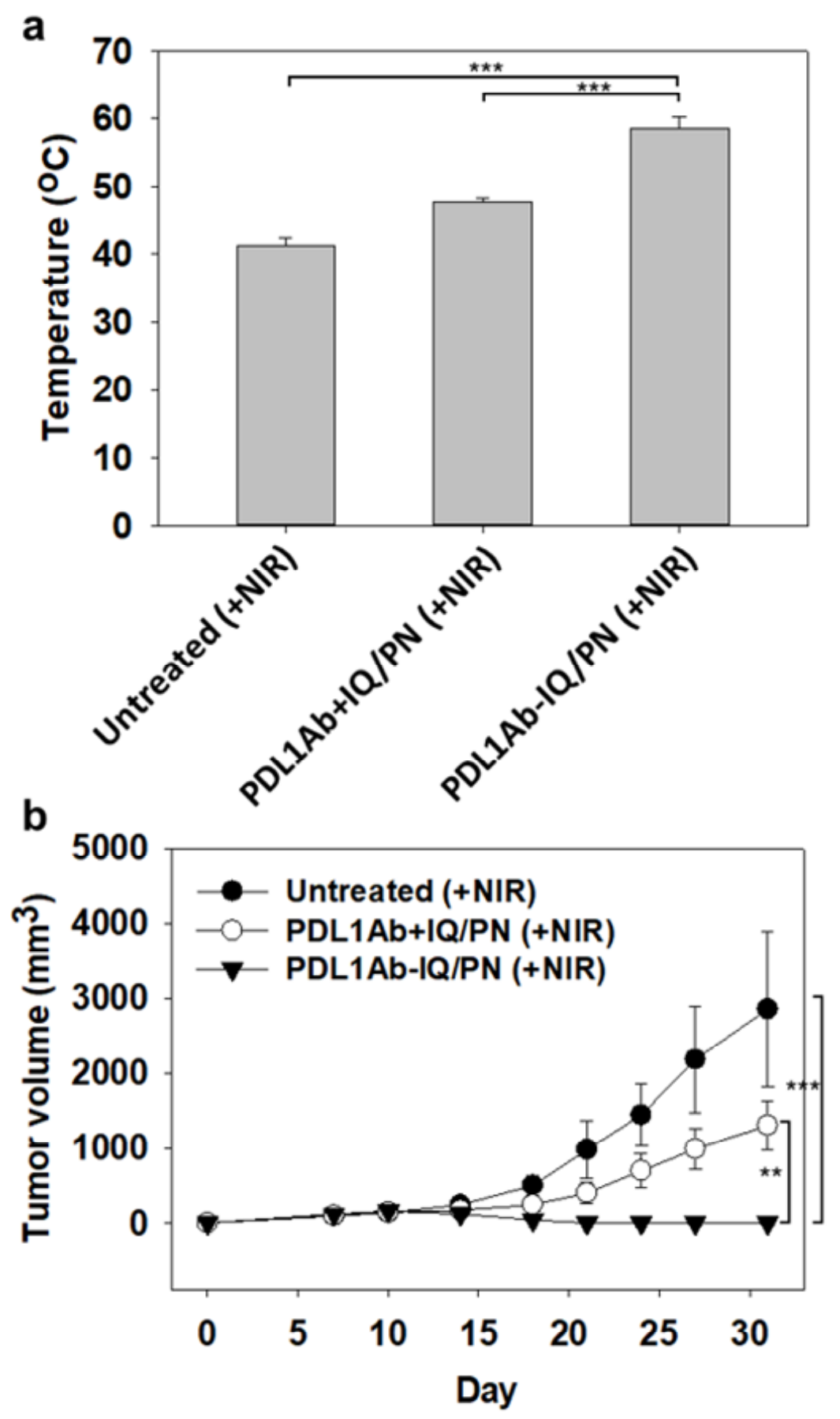

Figure S11. Photothermal anticancer efficacy of IQ/PN in combination with free PDL1Ab. CT26-bearing mice were intravenously injected with PDL1Ab-IQ/PNs or with IQ/PN plus free $\mathrm{PDL} 1 \mathrm{Ab}$ on day 7 after CT26 tumor inoculation. Mice were irradiated with an 808-nm laser on day 8. (a) Ten min after NIR irradiation, the temperature of tumor sites was monitored $(* * * P<$ 0.005). (b) The volumes of tumors were measured periodically until day 32 after tumor inoculation ( $* * * P<0.01 ; * * * P<0.005)$. 

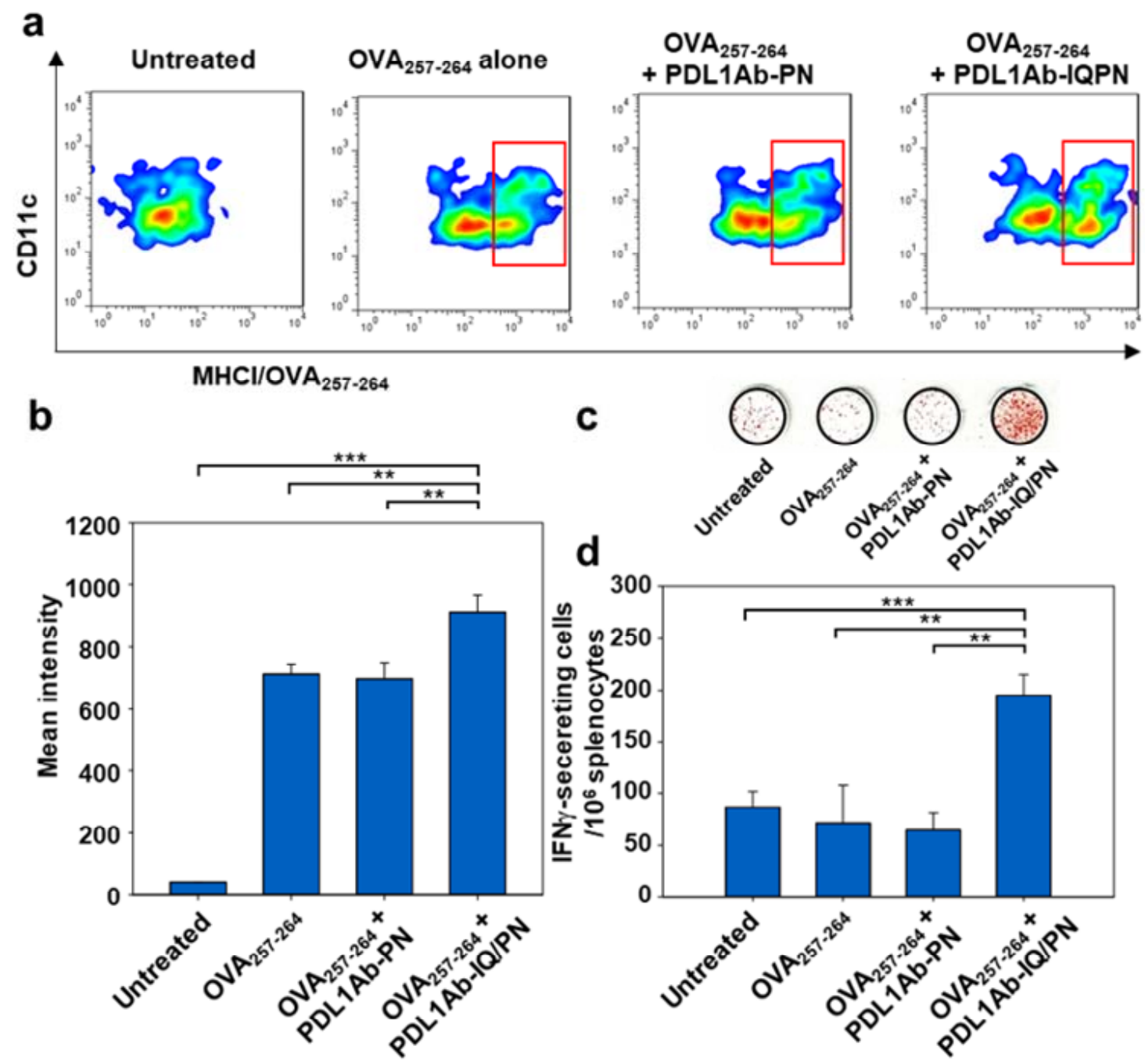

Figure S12. Antigen cross-presentation and T cell priming.

Splenocytes from C57BL mice were pulsed with $\mathrm{OVA}_{257-264}$ alone or with nanoformulations for 24 h. (a) Cross-presentation of $\mathrm{OVA}_{257-264}$ on CD11 $\mathrm{c}^{+}$dendritic cells was detected by staining with PE-anti-mouse $\mathrm{H}-2 \mathrm{~Kb} / \mathrm{OVA}_{257-264}$ antibody which specifically reacted with $\mathrm{OVA}_{257-264}$ bound to $\mathrm{H}-2 \mathrm{~Kb}$ of $\mathrm{MHC}$ class I using flow cytometry. (b) Quantification of the median fluorescence intensity of PE-conjugated anti-mouse $\mathrm{H}-2 \mathrm{~Kb} / \mathrm{OVA}_{257-264}$ antibody which specifically reacted with $\mathrm{OVA}_{257-264}$ bound to $\mathrm{H}-2 \mathrm{~Kb}$ of MHC class I. (c) Splenocytes from mice immunized with $\mathrm{OVA}_{257-264}$ and various $\mathrm{PN}$ formulations were isolated and re-stimulated with the OVA antigen peptide. Activation of $\mathrm{T}$ cells was tested by measuring IFN- $\gamma$ using an ELISPOT assay. (d) Quantification of IFN- $\gamma$ ELISPOT-positive cells. $(* * P<0.01$; $* * * P<$ $0.005)$. 


\section{a}
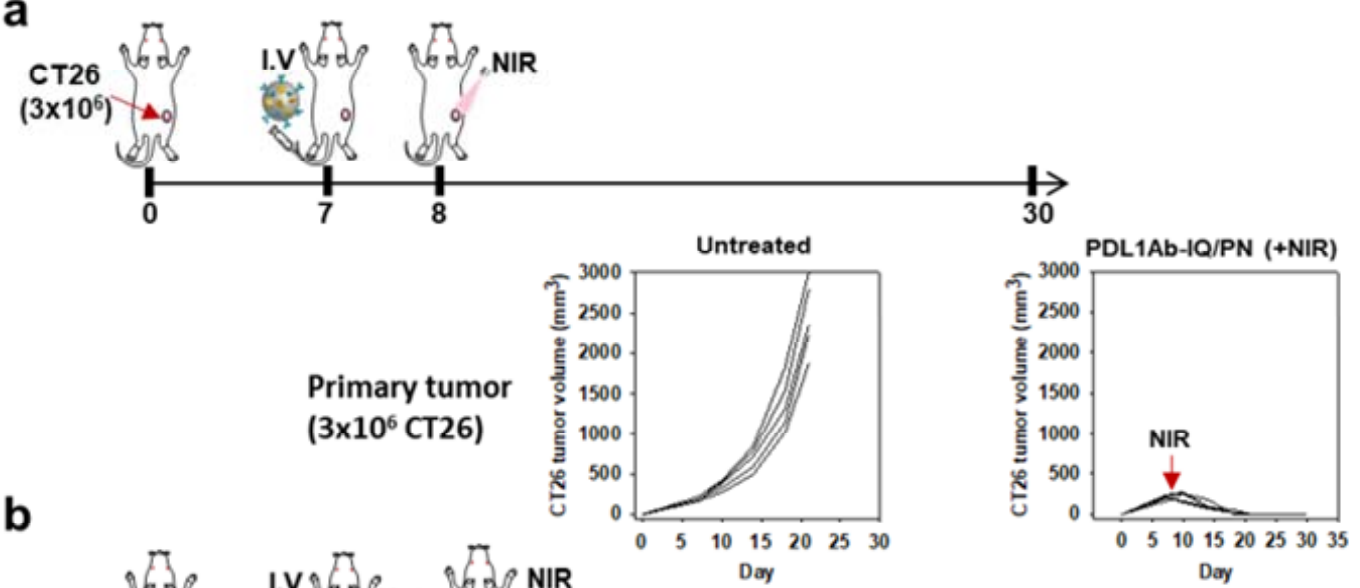

b

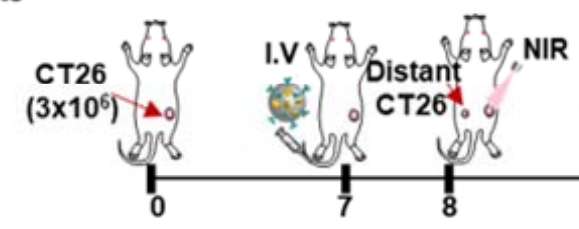

PDL1Ab (I.P)

Day

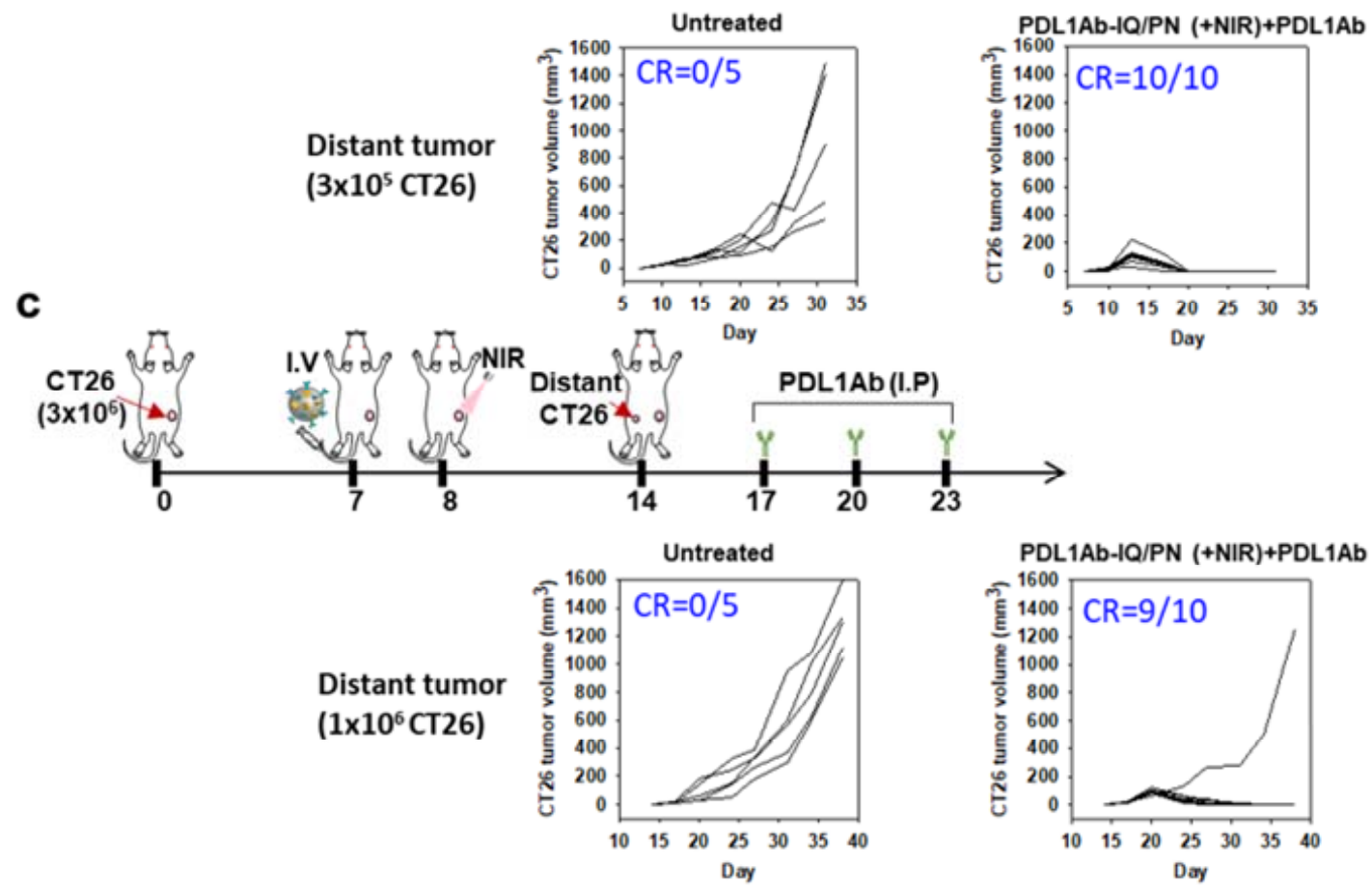

Figure S13. Effect of inoculating tumor cell number on the anticancer efficacy of PDL1AbIQ/PN.

(a) Each mouse was subcutaneously inoculated with $3 \times 10^{6}$ CT26 primary tumor cells. CT26bearing mice were intravenously injected with PDL1Ab-IQ/PN on day 7 and irradiated with an 808 -nm laser on day 8. (b) On the same day, mice were inoculated with $3 \times 10^{5}$ CT26 cells at a secondary site distant from the primary tumor. (c) On day 14, mice were inoculated with $1 \times 10^{6}$ CT26 cells at a secondary site distant from the primary tumor CT26 tumor. 


\section{Supplementary Video file}

Please, open the separately attached Supplementary Video File 1.

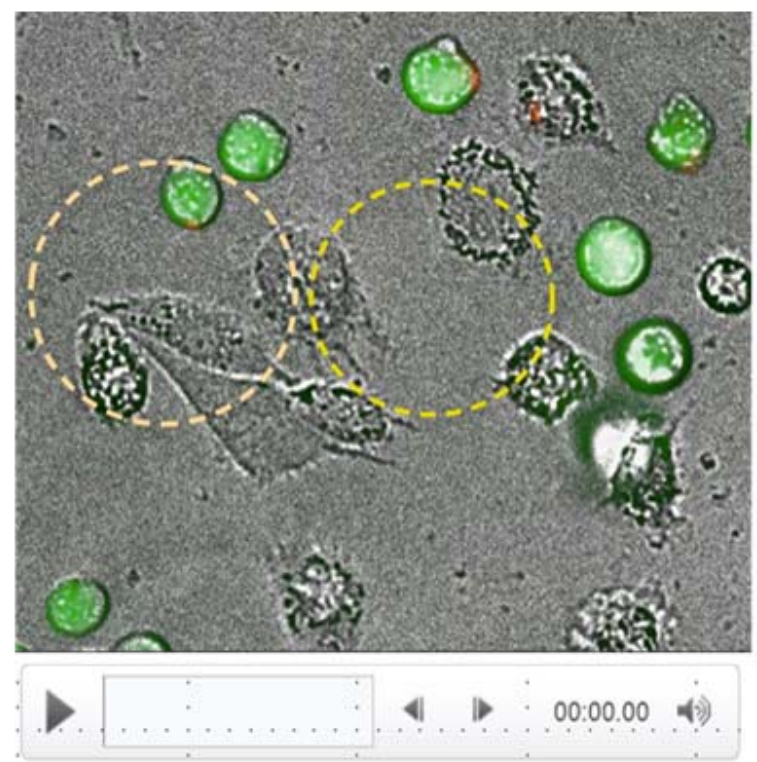

(Please, place cursor on each image for playing video file)

Video File S1. Real-time recording of phagocytosis of PDL1Ab-IQ/PN-treated CT26 cells (green) by BMDCs (red) (Video file).

CT26 cells (green) treated with PDL1Ab-IQ/PN and NIR-irradiated were co-cultured with BMDCs (red). The light or dark yellow dotted circles indicate DC phagocytosis. Co-cultures were immediately observed using an automated live-cell imaging system. 
Please, open the separately attached Supplementary video file 2.
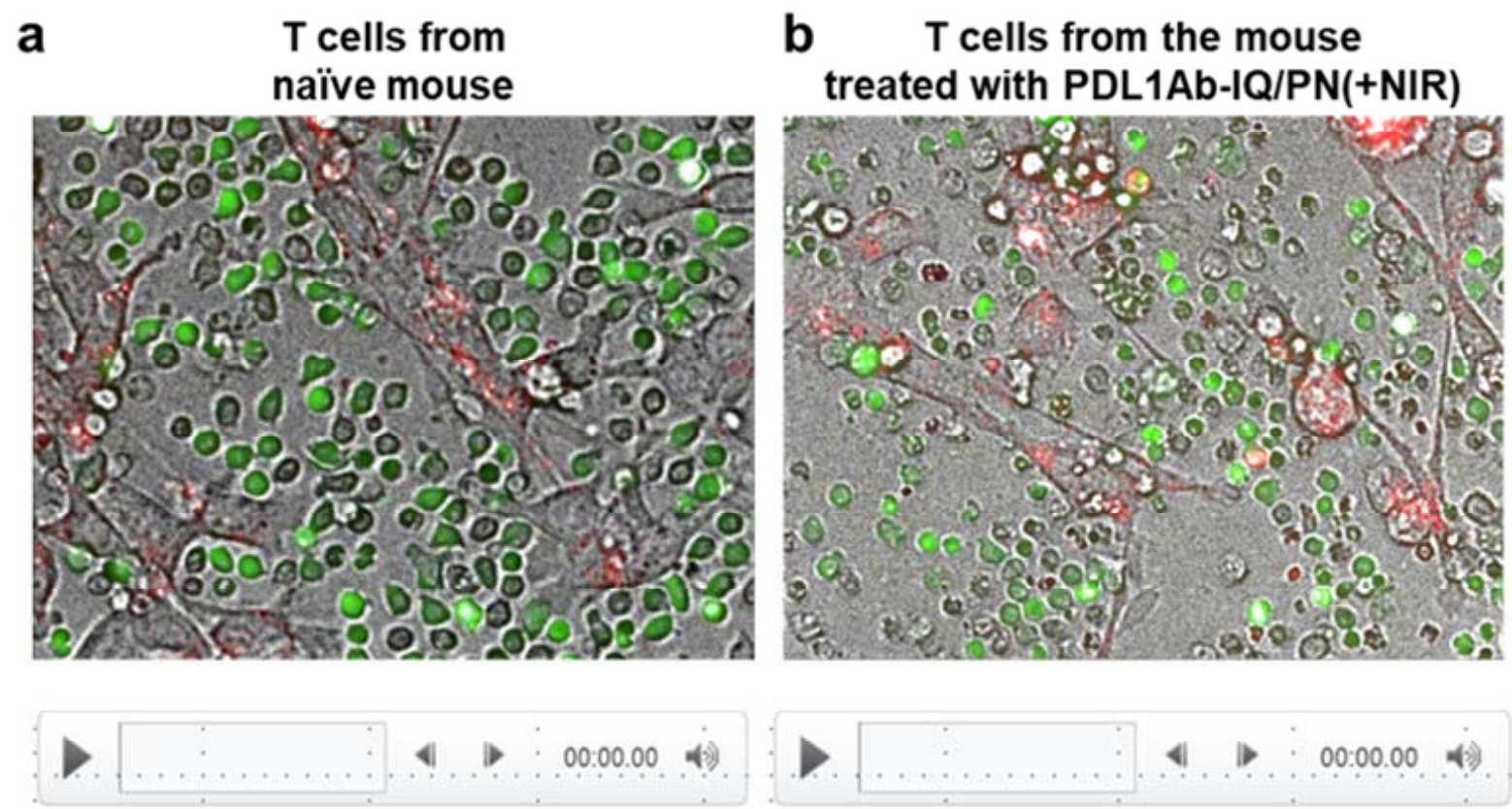

(Please, place cursor on each image for playing video file)

Video File S2. Real-time recording of the CT26 cell-killing effect of T cells (Video file).

CT26 cells (red) were co-cultured with splenocyte-derived T cells (green) from naïve mice (a) or CT26 tumor-survived mice treated with PDL1Ab-IQ/PNs and NIR-irradiated (b). Co-cultures were immediately observed using an automated live-cell imaging system. 